\title{
THE NONEXISTENCE OF GLOBALLY STABLE FORMS
}

\author{
MARTIN GOLUBITSKY AND DAVID TISCHLER ${ }^{1}$
}

\begin{abstract}
It is shown that on a closed manifold there are no globally stable differential forms.
\end{abstract}

A number of people have worked on the problem of the stability of differential forms. Martinet [1] has inspected the singularities and stability of germs of $p$-forms. His definition for stability is the following: a germ of a $p$ form $\omega$ is stable if for every nearby germ $\omega^{\prime}$ there is a germ of a diffeomorphism $f$ such that $f^{*} \omega^{\prime}=\omega$. In this paper Martinet computes some examples of stable germs. The stability of globally defined closed differential forms where the nearby forms $\omega^{\prime}$ are allowed to vary only within the cohomology class of $\omega$ have been studied by Moser [2], Chatelet and Rosenberg [3], and others.

A very tempting idea-given Martinet's sucess-is to try to find globally stable forms on a compact manifold $M$ using the following:

Definition 1. A $p$-form $\omega$ on a manifold $X$ is stable if there is a neighborhood $\Omega$ of $\omega$ in the $C^{\infty}$ topology on $p$-forms such that if $\omega^{\prime}$ is in $\Omega$, then there is a diffeomorphism $f: X \rightarrow X$ such that $f^{*} \omega^{\prime}=\omega$.

Unfortunately, this definition of stability for $p$-forms does have problems, for a little thought shows that there are obstructions to the existence of globally defined stable forms. In fact we show:

THEOREM 2. Using this definition of global stability, there do not exist globally stable forms on compact manifolds.

The authors wish to thank Mike Shub and Victor Guillemin for helpful conversations.

Our arguments fall into two classes: first we show that in a large number of cases of $p$-forms on $n$-manifolds even local stability for germs in the sense of Martinet is not possible; second, in the remaining cases there are global invariants which obstruct the existence of stable forms.

LEMMA 3. On an n-manifold where $n \geqslant 10$, there exist no locally or globally stable p-forms where $3 \leqslant p \leqslant n-3$. The same is true for 4 - or 5-forms on 9manifolds.

First, some notation. Let $\operatorname{Diff}(X)$ denote the group of $C^{\infty}$ diffeomorphisms

Received by the editors December 30, 1974 and, in revised form, October 27, 1975.

AMS (MOS) subject classifications (1970). Primary 58A10; Secondary 57D45.

${ }^{1}$ Research partially supported by NSF Contract no. GP 43524 and by a Faculty Research Grant of CUNY. 
on $X$, let $\Lambda^{p}=\Lambda^{p}\left(T^{*} X\right)$ denote the space of $C^{\infty} p$-forms on $X$, and let $\Lambda_{x}^{p}$ be the fiber of the bundle $\Lambda^{p}\left(T^{*} X\right)$ over the point $x$ in $X$.

Proof. Suppose $\omega$ is a stable $p$-form; then the orbit $\Phi_{\omega}$ of the action of $\operatorname{Diff}(X)$ on $\Lambda^{p}$ through $\omega$ given by pull-back is open. Let $V_{x}(\omega)=\left\{\omega^{\prime}(x)\right.$ $\left.\in \Lambda_{x}^{p} \mid \omega^{\prime} \in \Phi_{\omega}\right\}$ for $x$ in $X$. Then $V_{x}(\omega)$ is open in $\Lambda_{x}^{p}$.

Next choose a neighborhood $U$ of $x$ in $X$ over which the bundle $\Lambda^{p}$ is trivial. Using this trivialization one has a projection map $\pi: \Lambda^{p} \mid U \rightarrow \Lambda_{x}^{p}$. Consider the map

$$
\psi: U x G l\left(T_{x} X\right) \rightarrow \Lambda_{x}^{p}
$$

defined by $(y, A) \rightarrow \pi\left(A^{*} \omega\right) y$ where $T_{x} X$ is the tangent space to $X$ at $x$ and where $A$ acts on $T_{y} X$ using the trivialization. It is easy to show that the image of $\psi$ contains $V_{x}$. So if $\omega$ is stable, then $\operatorname{Im} \psi$ is open and

$$
\operatorname{dim}\left(U x G l\left(T_{x} X\right)\right) \geqslant \operatorname{dim} \Lambda_{x}^{p} .
$$

So we have that $n+n^{2} \geqslant\left(\begin{array}{l}n \\ p\end{array}\right)$; but this cannot happen when $n \geqslant 10$ and $3 \leqslant p \leqslant n-3$ or when $n=9$ and $p=4$ or 5 .

Now we attack the global problem.

Definition 4. A mapping $\varphi$ of $p$-forms on $X$ to densities on $X$ is destabilizing if for every $p$-form $\omega$

$$
\int_{X} \varphi\left(f^{*} \omega\right)=\int_{X} \varphi(\omega) \text { for all } f \text { in } \operatorname{Diff}(X),
$$

and (2) there is a curve $\omega_{t}$ of $p$-forms with $\omega_{0}=\omega$ and

$$
\int_{X} \varphi(\omega) \neq \int_{X} \varphi\left(\omega_{t}\right) \text { for all } t \neq 0 .
$$

Note that since $\varphi(\omega)$ is a density as opposed to an $n$-form, $\int_{X} \varphi(\omega)$ is well defined whether or not $X$ is orientable.

LeMma 5. If there exists a destabilizing mapping on p-forms, then there are no stable p-forms.

Proof. Obvious.

Lemma 6. In all cases not included in Lemma 3 where $p>0$ there exist destabilizing mappings $\varphi$.

Proof. Define $\varphi$ in the given cases as follows:

$$
\begin{array}{ll}
p=n & \varphi(\omega)=|\omega|, \\
p=2 n-1 & \varphi(\omega)=|d \omega|, \\
p=1, \quad n=2 k & \varphi(\omega)=\left|(d \omega)^{k}\right|, \\
p=1, \quad n=2 k+1 & \varphi(\omega)=\left|\omega \wedge(d \omega)^{k}\right|, \\
p=2, \quad n=2 k & \varphi(\omega)=\left|\omega^{k}\right|, \\
p=2, \quad n=2 k+1 & \varphi(\omega)=\left|\omega^{k-1} \wedge d \omega\right|,
\end{array}
$$




$$
\begin{array}{ll}
p=3, n=7 & \varphi(\omega)=|\omega \wedge d \omega|, \\
p=3, \quad n=8 & \varphi(\omega)=\left|(d \omega)^{2}\right|, \\
p=4, \quad n=8 & \varphi(\omega)=\left|\omega^{2}\right| .
\end{array}
$$

In these cases, condition (1) of Definition 4 is obvious. For condition (2) we see that if $\varphi(\omega) \neq 0$, we can take $\omega_{t}=(t+1) \omega$; while if $\varphi(\omega)=0$ we need a short argument. For example, in the case $p=1, n=2 k$ there is a 1-form $\sigma$ such that $(d \sigma)^{k} \neq 0$. This is obviously true locally; extend the form to a global one in some convenient fashion. Then take $\omega_{t}=\omega+t \sigma$ and note that $\left(d \omega_{t}\right)^{k}=t(d \omega)^{k-1} \wedge d \sigma+\cdots+t^{k}(d \sigma)^{k}$ since $\varphi(\omega)=\left|(d \omega)^{k}\right|=0$. There is a first nonzero term since $(d \sigma)^{k} \neq 0$ and for small $t$ this term dominates. So it is impossible for $\varphi\left(\omega_{t}\right)=0$ for all $t$ small and $\int_{X} \varphi\left(\omega_{t}\right) \neq 0$. Note we use the facts that $\varphi \not \equiv 0$ and $\varphi(t \omega)=|t|^{l} \varphi(\omega)$ for some number $l \neq 0$ to construct the curve $\omega_{t}$ which shows that $\varphi$ is destabilizing. For the other cases listed above these facts are clear. For the following cases a similar proof will work.

The cases that remain are

$$
(p, n)=(n-2, n),(3,6),(3,9),(4,7),(5,8), \text { or }(6,9) .
$$

For these we need a different argument. First, we do the construction of $\varphi$ for $(n-2)$-forms on an $n=2 k$-manifold $X$.

Let $V$ be a vector space and let $\Lambda^{n-2}(V)$ be the $(n-2)$-forms in the Grassmann algebra on $V$. Let

$$
\Delta: \Lambda^{n-2}\left(V^{*}\right) \rightarrow \otimes_{i=1}^{k} \Lambda^{n-2}\left(V^{*}\right)
$$

be the diagonal map, let

$$
P: \Lambda^{n}\left(V^{*}\right) \otimes \Lambda^{n}(V) \rightarrow R
$$

be the natural pairing, and let

$$
\wedge: \bigotimes_{i=1}^{k} \Lambda^{2}(V) \rightarrow \Lambda^{2 k}(V)
$$

be the linear map induced by wedge product.

Now suppose $X$ is a $2 k$-manifold. Then we can consider the following sequence of maps:

$$
\begin{aligned}
& \Lambda^{n-2}\left(T^{*} X\right) \stackrel{\Delta}{\longrightarrow} \bigotimes_{i=1}^{k} \Lambda^{n-2}\left(T^{*} X\right) \cong \bigotimes_{i=1}^{k} \operatorname{Hom}\left(\Lambda^{2}\left(T^{*} X\right), \Lambda^{n}\left(T^{*} X\right)\right) \\
& \cong \bigotimes_{i=1}^{k} \Lambda^{n}\left(T^{*} X\right) \otimes \bigotimes_{i=1}^{k} \Lambda^{2}(T X) \stackrel{i d \otimes \Lambda}{\longrightarrow} \bigotimes_{i=1}^{k-1} \Lambda^{n}\left(T^{*} X\right) \otimes \Lambda^{n}\left(T^{*} X\right) \otimes \Lambda^{n}(T X) \\
& \stackrel{i d \otimes P}{\longrightarrow} \bigotimes_{i=1}^{k-1} \Lambda^{n}\left(T^{*} X\right)
\end{aligned}
$$


Call the composite map $\sigma$. Then given an $(n-2)$-form $\omega, \sigma(\omega)$ is a section of the one-dimensional bundle $\otimes_{i=1}^{k-1} \Lambda^{n}\left(T^{*} X\right)$. The transition functions for this bundle are related by (determinant $)^{k-1}$. So taking the $(k-1)$ st root of $|\sigma(\omega)|$ gives a section of the one-dimensional bundle whose transition functions are related by |determinant|, in other words, a density on $X$. So we set $\varphi(\omega)$ $=|\sigma(\omega)|^{1 /(k-1)}$. Since all the maps used to define $\sigma$ are natural, $\varphi$ satisfies (1) of Definition 4. It is easy to see that $\varphi(t \omega)=|t|^{k /(k-1)} \varphi(\omega)$. To see that $\varphi$ is destabilizing, we need only show that $\varphi$ is not identically zero. This reduces to showing that $\wedge: \otimes_{i=1}^{k} \Lambda^{2}(T X) \rightarrow \Lambda^{2 k}(T X)$ is not zero; but this is clear since the image of the standard symplectic 2-coform (in any basis) under $\wedge$ is not zero.

The remaining cases are all similar. To define $\sigma$ for $(n-2)$-forms on $n=(2 k+1)-$ manifolds, consider the following:

$$
\begin{aligned}
\Lambda^{n-2}\left(T^{*} X\right) & \stackrel{\Delta \otimes d}{\longrightarrow} \bigotimes_{i=1}^{k} \Lambda^{n-2}\left(T^{*} X\right) \otimes \Lambda^{n-1}\left(T^{*} X\right) \\
& \cong \bigotimes_{i=1}^{k} \operatorname{Hom}\left(\Lambda^{2}\left(T^{*} X\right), \Lambda^{n}\left(T^{*} X\right)\right) \otimes \operatorname{Hom}\left(\Lambda^{1}\left(T^{*} X\right), \Lambda^{n}\left(T^{*} X\right)\right) \\
& \cong \bigotimes_{i=1}^{k+1} \Lambda^{n}\left(T^{*} X\right) \otimes \bigotimes_{i=1}^{k} \Lambda^{2}(T X) \otimes \Lambda^{1}(T X) \\
& \stackrel{i d \otimes \Lambda}{\longrightarrow} \bigotimes_{i=1}^{k} \Lambda^{n}\left(T^{*} X\right) \otimes \Lambda^{n}\left(T^{*} X\right) \otimes \Lambda^{n}(T X) \\
& \stackrel{i d \otimes P}{\longrightarrow} \bigotimes_{i=1}^{k} \Lambda^{n}\left(T^{*} X\right) .
\end{aligned}
$$

As before, take $\varphi(\omega)=|\sigma(\omega)|^{1 / k}$ and note that $\varphi(t \omega)=|t|^{(k+1) / k} \varphi(\omega)$. Since $\varphi \not \equiv 0, \varphi$ is also a destabilizing mapping; so there are no stable codimension 2-forms.

For $(p, n)=(3,6)$ or $(5,8)$, we take $\varphi(\omega)=\varphi(d \omega)$. Note that $d \omega$ is a codimension 2-form on an even-dimensional manifold. This trick will not work for codimension 3-forms on an odd-dimensional manifold for the construction of $\sigma$ in this case already includes the exterior derivative $d$ and the composite map $\sigma \circ d$ is then identically zero.

For $(p, n)=(4,7)$ we construct $\sigma$ as follows:

$$
\begin{aligned}
& \Lambda^{4}\left(T^{*} X\right) \stackrel{i d \otimes d \otimes d}{\longrightarrow} \Lambda^{4}\left(T^{*} X\right) \otimes \underset{i=1}{\otimes} \Lambda^{5}\left(T^{*} X\right) \\
& \stackrel{i d \otimes \Lambda}{\longrightarrow} \stackrel{\otimes}{i=1}_{i}^{7} \Lambda^{*}\left(T^{*} X\right) \otimes \Lambda^{3}(T X) \otimes \Lambda^{4}(T X)
\end{aligned}
$$

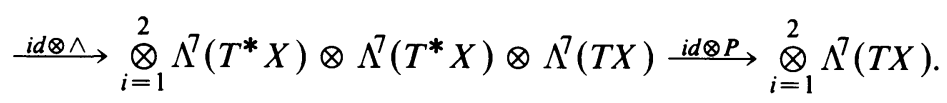

Set $\varphi(\omega)=|\sigma(\omega)|^{1 / 2}$. For $(p, n)=(6,9)$, define $\sigma$ by: 


$$
\begin{aligned}
\Lambda^{6}\left(T^{*} X\right) & \stackrel{i d \otimes d \otimes d \otimes d}{\longrightarrow} \Lambda^{6}\left(T^{*} X\right) \otimes \bigotimes_{i=1}^{3} \Lambda^{7}\left(T^{*} X\right) \\
& \cong \bigotimes_{i=1}^{4} \Lambda^{9}\left(T^{*} X\right) \otimes \Lambda^{3}(T X) \otimes \bigotimes_{i=1}^{3} \Lambda^{2}(T X) \\
& \stackrel{i d \otimes \wedge}{\longrightarrow} \bigotimes_{i=1}^{3} \Lambda^{9}\left(T^{*} X\right) \otimes \Lambda^{9}\left(T^{*} X\right) \otimes \Lambda^{9}(T X) \stackrel{i d \otimes P}{\longrightarrow} \otimes_{i=1}^{3} \Lambda^{9}\left(T^{*} X\right) .
\end{aligned}
$$

Set $\varphi(\omega)=|\sigma(\omega)|^{1 / 3}$.

Finally, for $(p, n)=(3,9)$ construct $\sigma$ as follows:

$$
\Lambda^{3}\left(T^{*} X\right) \rightarrow \Lambda^{7}\left(T^{*} X\right) \otimes \Lambda^{7}\left(T^{*} X\right) \otimes \Lambda^{4}\left(T^{*} X\right)
$$

(given by $\omega \rightarrow(\omega \wedge d \omega) \otimes(\omega \wedge d \omega) \otimes d \omega)$

$$
\begin{aligned}
& \cong \bigotimes_{i=1}^{3} \Lambda^{9}\left(T^{*} X\right) \otimes \bigotimes_{i=1}^{2} \Lambda^{2}(T X) \otimes \Lambda^{5}(T X) \\
& \stackrel{i d \otimes \Lambda}{\longrightarrow} \bigotimes_{i=1}^{3} \Lambda^{9}\left(T^{*} X\right) \otimes \Lambda^{9}(T X) \stackrel{i d \otimes P}{\longrightarrow} \otimes_{i=1}^{2} \Lambda^{9}\left(T^{*} X\right) .
\end{aligned}
$$

Set $\varphi(\omega)=|\sigma(\omega)|^{1 / 2}$. Q.E.D.

Proof of Theorem 2. Lemmas 3.5 and 6 show that there are no stable $p$ forms when $p>0$. To complete the proof note that 0 -forms are just functions and that the action of $\operatorname{Diff}(X)$ on functions given by pull-back preserves critical values. Since critical values can always be perturbed, 0 -forms are never stable. Q.E.D.

To complete our discussion, we note that global stability for closed forms could be defined as in Definition 1, if closed $p$-form is substituted for $p$-form throughout. This definition is just as bad as Definition 1, for Lemma 3 is still valid and Lemma 6 is valid in any case where the destabilizing mapping was defined without the use of exterior differentiation. Also, it is easy to show that if a closed $p$-form on $X$ is stable, then the $p$ th cohomology on $X$ with real coefficients is zero.

\section{BIBLIOGRAPHY}

1. J. Martinet, Sur les singularités des formes différentielles, Ann. Inst. Fourier (Grenoble) 20 (1970), fasc. 1, 95-178. MR 44 \#333.

2. J. K. Moser, On the volume elements on a manifold, Trans. Amer. Math. Soc. 120 (1965), 286-294. MR 32 \#409.

3. G. Chatelet and H. Rosenberg, Conjugacy of closed non-vanishing 1-forms (to appear).

Department of Mathematics, Queens College of the City University of New York, Flushing, NeW YoRK 11367 